\title{
Evaluating the Performance of Syndromic Surveillance System using High-fidelity Outbreak Simulations
}

\author{
Tao Tao*1, Qi Zhao', Shaofa Nie², Lars Palm³, Vinod K. Diwan and Biao Xu \\ ${ }^{1}$ School of Public Health, Fudan University, Shanghai, China; ${ }^{2}$ Department of Epidemiology and Biostatistics, School of Public \\ Health, Tongji Medical College, Huazhong University of Science and Technology, Wuhan, China; ${ }^{3}$ Future Position X (FPX), Gävle, \\ Sweden; ${ }^{4}$ Division of Global Health (IHCAR), Department of Public Health Sciences, Karolinska Institutet, Stockholm, Sweden
}

\section{Objective}

Our study aimed to conduct high-fidelity simulations based on real-world outbreaks for evaluating the performance of syndromic surveillance system.

\section{Introduction}

The evaluation of outbreak detection performance remained a major challenge to every syndromic surveillance system. Owing to the scarcity and uncertainty of infectious disease outbreaks in the real world, simulated outbreak datasets have been commonly used by scholars for performance evaluation. Although this method was powerful in estimating the performance of syndromic surveillance across a variety of outbreak scenarios, the inevitable differences between simulation and authentic outbreak event limited its external validity.

\section{Methods}

Since April 2012, a hospital-based syndromic surveillance system (ISSC) has been implemented in two rural counties of Jiangxi Province, China. From ISSC database we exported 10 months' syndromic data collected between May 1st 2012 and February 28th 2013 (304 days) as the baseline for performance evaluation. Then, we reviewed the archives of epidemiological investigation in local CDCs to search for confirmed infectious or non-infectious disease outbreaks occurred during the 10 months' period as candidate spike for simulation. For each outbreak found in ISSC system, its epidemic curve was extracted and injected day by day to the non-outbreak baseline surveillance data of the same unit (e.g., an outbreak found in one hospital will only be injected into the baseline of this hospital), to construct "high-fidelity" simulations. EARS' C1, C2 and C3 algorithms were run among simulated datasets to calculate the timeliness of outbreak detection ([detection delay / duration of outbreak] $* 100 \%$ ) under 5 different levels of specificity (defined as the proportion of days that do not trigger alarms in non-outbreak period).

\section{Results}

Three outbreak events caused by different diseases (varicella, food poisoning and influenza) were captured by ISSC system during the study period. The duration of outbreak was one day for food poisoning, 6 days for influenza and 37 days for varicella (Figure 1). Simulation results showed that the timeliness of outbreak detection using $\mathrm{C} 1$ algorithm varied from $8 \%$ of outbreak duration (detecting varicella outbreak using "First \& Repeat visit" group under a $82 \%$ specificity) to $90 \%$ of outbreak duration (detecting foodborne outbreak using "All symptom" group under a $98 \%$ specificity), and the detection timeliness of varicella outbreak was higher than foodborne $\&$ influenza outbreaks. Different syndromic groups presented distinct performances in outbreak detection (Figure 2). The timeliness of all syndromic groups decreased significantly as the specificity level increased, and the $\mathrm{C} 1 \& \mathrm{C} 2$ algorithms outperformed $\mathrm{C} 3$ algorithm in outbreak detection among all circumstances.

\section{Conclusions}

High-fidelity simulation revealed that syndromic surveillance system was capable to detect the 3 disease outbreaks tested at an early stage, but the practical performance was to a great extent affected by the type and magnitude of outbreak event, the selection of syndromic groups for monitoring, the detection algorithm introduced in the system, and the preferred false alarm rate in real-time surveillance. Our study results indicated the importance of exploring disease-specific syndromic groups for outbreak detection.

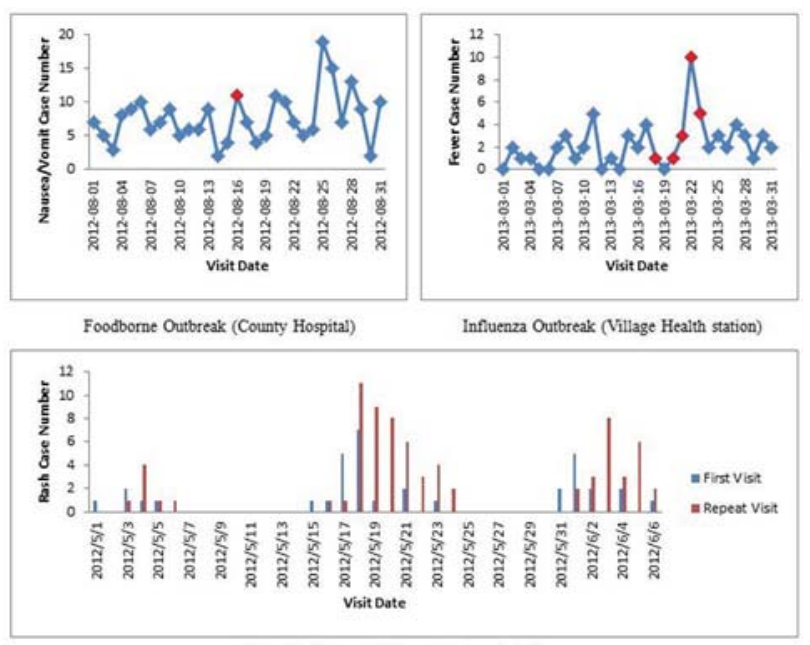

Varicella Outbreak (Village Health station)

Figure 1 Outbreak manifestations in the present of background data

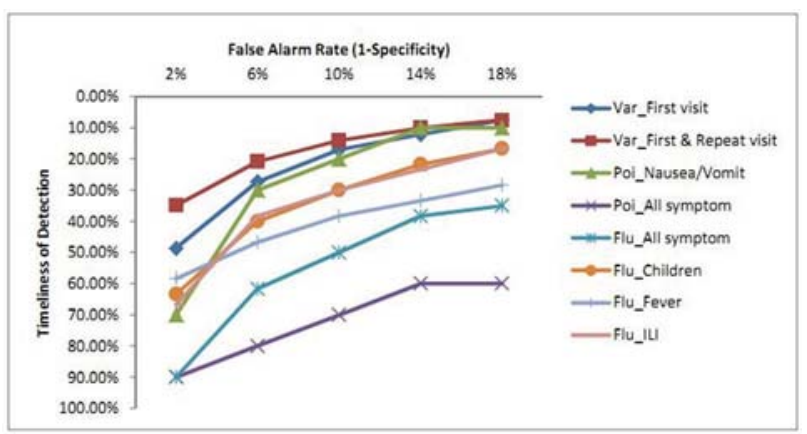

Figure 2 AMOC curves of outbreak detection performance using different syndromic groups (High-fidelity simulation with $\mathrm{Cl}$ algorithm, Var=varicella, Poi=food poisoning, Flu=Influenza)

\section{Keywords}

simulation; performance; timeliness

\section{Acknowledgments}

This study was funded by European Union's Seventh Framework Programme ([FP7/2007-2013] [FP7/2007-2011]) under grant agreement no. [241900].

*Tao Tao

